\title{
Cellular Automata Segmentation of Brain Tumors on Post Contrast MR Images
}

\author{
Andac Hamamci ${ }^{1}$, Gozde Unal ${ }^{1}$, Nadir Kucuk ${ }^{2}$, and Kayihan Engin ${ }^{2}$ \\ ${ }^{1}$ Faculty of Engineering and Natural Sciences, Sabanci University, Istanbul, Turkey \\ gozdeunal@sabanciuniv.edu \\ ${ }^{2}$ Department of Radiation Oncology, Anadolu Medical Center, Kocaeli, Turkey
}

\begin{abstract}
In this paper, we re-examine the cellular automata(CA) algorithm to show that the result of its state evolution converges to that of the shortest path algorithm. We proposed a complete tumor segmentation method on post contrast T1 MR images, which standardizes the VOI and seed selection, uses CA transition rules adapted to the problem and evolves a level set surface on CA states to impose spatial smoothness. Validation studies on 13 clinical and 5 synthetic brain tumors demonstrated the proposed algorithm outperforms graph cut and grow cut algorithms in all cases with a lower sensitivity to initialization and tumor type.
\end{abstract}

\section{Introduction}

Segmentation of tumors on medical images is not only of high interest in serial treatment monitoring of "disease burden" in oncologic imaging, but also gaining popularity with the advance of image guided surgical approaches [1]. Outlining the tumor contour is a major step in planning spatially localized radiotherapy (e.g. Cyberknife, iMRT) which is done manually on post contrast T1 MRI in current clinical practice. On T1 images acquired after administration of a contrast agent (gadolinium), blood vessels and the parts of the tumor, where the contrast can pass the blood-brain barrier are observed as hyper intense areas.

Region-based active contour models are widely used in image segmentation 2]. In general, these region-based models have several advantages over gradientbased techniques for segmentation, including greater robustness to noise. However, classical snakes had the problem of being "only as good as their initialization", even when using level-set snakes in 3D. Because the tumor class does not have a strong spatial prior, many small structures, mainly blood vessels, are classified as tumor as they also enhance with contrast. Ho et.al. used fuzzy classification of pre and post contrast T1 images to obtain a tumor probability map to evolve a level-set snake [3. Liu et.al. have adapted the fuzzy connectedness framework for tumor segmentation by constructing a rectangular volume of interest selected through identifying the first and last slice of the tumor and specifying a set of voxels in the tumor region [4].

Interactive algorithms have become popular for image segmentation problem in recent years. Graph based seeded segmentation framework has been generalized such that graph cuts (GC) [5], random walker (RW) [6], shortest paths,

T. Jiang et al. (Eds.): MICCAI 2010, Part III, LNCS 6363, pp. $137-146,2010$.

(C) Springer-Verlag Berlin Heidelberg 2010 
and power watersheds [7] have been interpreted as special cases of a general seeded segmentation algorithm, which solves a minimization problem involving a graph's edge weights constrained by adjacent vertex variables or probabilities. In [8, the connection between GC, RW, and shortest paths was shown to depend on different norms: $L_{1}(\mathrm{GC}) ; L_{2}(\mathrm{RW}) ; L_{\infty}$ (shortest paths), in the energy that is optimized. Although it was reported that the shortest paths and RW produce relatively more seed-dependent results, it can be argued that the global minimum of an image segmentation energy is worth as good as the ability of its energy to capture underlying statistics of images [9], and a local minimum may produce a solution closer to the ground truth than that of a global minimum. Hence, with good prior information provided as in the case of a seeded image segmentation problem, efficiently finding a good local minima becomes meaningful and worthwhile.

On the other hand, cellular automata (CA) algorithm motivated biologically from bacteria growth and competition, is based on a discrete dynamic system defined on a lattice, and iteratively propagates the system states via local transition rules. It was first used by Vezhnevets et.al. [10] (grow-cuts) for image segmentation, which showed the potential of the $\mathrm{CA}$ algorithm on generic medical image problems.

In this paper, we re-examine the $\mathrm{CA}$ algorithm to establish the connection of the CA-based segmentation to the graph-theoretic methods to show that the iterative CA framework converges to the shortest path algorithm, for the first time, to our knowledge. Next, as our application is in the clinical radiotherapy planning, where manual segmentation of tumors are carried out on CT fused post contrast T1-MR images by a radio-oncology expert, we modify the CA segmentation towards the nature of the tumor properties undergoing radiation therapy by adapting relevant transition rules. Finally, a smoothness constraint using level set active surfaces is imposed over the resulting CA states. We present our framework for brain tumor segmentation in Section 2, and demonstrate its performance via validation studies on both synthetic, and radiation therapy planning expert-segmented data sets in Section 3, followed by discussions and conclusions.

\section{Method}

\subsection{Cellular Automata: Its Connection to Graph Theoretic Methods}

A graph consists of a pair $G=(V, E)$ with vertices (nodes) $v \in V$ and edges $e \in$ $E \subseteq V \times V$. The weight of an edge, $e_{i j}$, is denoted by $w_{i j}$ and is assumed here to be nonnegative and undirected (i.e., $w_{i j}=w_{j i}$ ). We will use closed neighborhood $N_{G}[v]$ where $v_{i} \in N_{G}\left(v_{i}\right)$. The edge weights are similarity measures calculated using measured data (e.g. voxel intensity) for vertices: $w_{i j}=f\left(I_{i}, I_{j}\right) \in(0,1]$ and self-similarity $w_{i i}=1$. State of a vertex $s\left(v_{i}\right)=s_{i}$ is specified with a real value $x\left(v_{i}\right)=x_{i} \in[0,1]$ and a label $l_{i} \in\{B G, F G, \cdots\}$ pair. Starting with 
initial states of vertices, in each iteration, vertices of graph $\mathrm{G}$ is updated by the following rule:

$$
l_{i}^{t+1}=l_{i^{*}}^{t} \text { and } x_{i}^{t+1}=w_{i^{*} i} x_{i^{*}}^{t} \text { where } i^{*}=\arg \max _{j \in N_{G}\left[v_{i}\right]} w_{j i} x_{j}
$$

Note that since the vertex itself is also included in its neighborhood, Eq. (1) also covers the static case:

$$
s_{i}^{t+1}=s_{i}^{t} \text { if } x_{i} \geq w_{j i} x_{j} \text { for } \forall v_{j} \in N_{G}\left[v_{i}\right] \backslash v_{i}
$$

Vertex states are initialized by user supplied seeds $p_{i} \in P$ such as:

$$
s^{0}\left(v_{i}\right)=\left(1, l\left(p_{i}\right)\right) \text { for } v_{i} \in P \text { and } s^{0}\left(v_{i}\right)=(0, \emptyset) \text { for } v_{i} \notin P
$$

This map converges since $\sum_{i} x_{i}$ is upper-bounded and monotonically increasing:

$$
\lim _{t \rightarrow \infty} s_{i}^{t+1}=s_{i}^{t} \text { for } \forall v_{i} \in V
$$

Now, let us derive some properties on the final map. Consider any vertex $v_{i}$ of a graph G, and assume that a latest update occurred on this vertex at time $t_{i}$. The vertex which updates $v_{i}$ is $v_{i^{*}}$. Final state for $v_{i}$ is:

$$
s_{i}^{t \geq t_{i}}=\left(w_{i^{*} i} x_{i^{*}}^{t_{i}}, l_{i^{*}}^{t_{i}}\right)
$$

If any update occurs on $v_{i^{*}}$ at time $t_{i^{*}} \geq t_{i}$ by $v_{i^{* *}}$, this should satisfy the condition:

$$
x_{i^{*}}^{t_{i^{*}}}=w_{i^{* *} i^{*}} x_{i^{* *}}^{t_{i^{*}}}>x_{i^{*}}^{t<t_{i^{*}}} \text { that gives } w_{i^{*} i} x_{i^{*}}^{t_{i^{*}}}>w_{i^{*} i} x_{i^{*}}^{t<t_{i^{*}}}
$$

However, this will also cause an update on $v_{i}$ at $t>t_{i^{*}}>t_{i}$, which violates the condition in (5). Then, at the converged map, there exists a neighbor $v_{i *}$ for each vertex $v_{i}$ such that:

$$
s_{i}=\left(w_{i^{*}} x_{i^{*}}, l_{i^{*}}\right)
$$

If we go one step further:

$$
s_{i^{*}}=\left(w_{i^{* *} i^{*}} x_{i^{* *}}, l_{i^{* *}}\right) \text { and } s_{i}=\left(w_{i^{*} i} w_{i^{* *} i^{*}} x_{i^{* *}}, l_{i^{* *}}\right)
$$

We can follow this path for any vertex until we reach a seed which is never updated:

$$
s\left(v_{i}\right)=\left(\prod_{\Omega\left(p_{i} \rightarrow v_{i}\right)} w_{j k}, l\left(p_{i}\right)\right)
$$

Therefore, this algorithm cuts the graph $G$ to independent subgraphs for each seed, consisting of spanning trees with seeds at root nodes.

If we set edge weights depending on similarity of image $\left(I: R^{3} \rightarrow R\right)$ neighborhoods as:

$$
w_{j k}=e^{-B\left\|\nabla_{j k} I\right\|}
$$


where $\left\|\nabla_{j k} I\right\|$ denotes a Euclidean norm on the difference between intensities of two adjacent vertices $v_{j}$ and $v_{k}$. Maximization the product of $w_{j k}$ 's along the path $\Omega$ becomes equivalent to minimization of the summation of $\left\|\nabla_{j k} I\right\|$ 's along the same path. $\sum_{\Omega\left(p_{i} \rightarrow v_{i}\right)}\left\|\nabla_{j k} I\right\|$ is a discrete approximation to a geodesic or shortest path between the seed $p_{i}$ to a voxel $v_{i}$. Each voxel is then assigned to the foreground label if there is a shorter path from that voxel to a foreground seed than to any background seed, where paths are weighted by image content. With this interpretation, cellular automata algorithm solves the shortest paths energy form formulated in [8].

The equivalence, which we showed, between CA updates by Eq. (11) and shortest path algorithm is illustrated in Fig. 1.

The main advantage of using CA algorithm is its ability to obtain a multilabel solution in a simultaneous iteration. Another advantage is that the local transition rules are simple to interpret, and it is possible to impose prior knowledge, specific to the problem, into the segmentation algorithm.

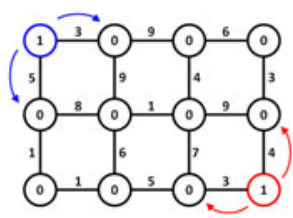

(a)

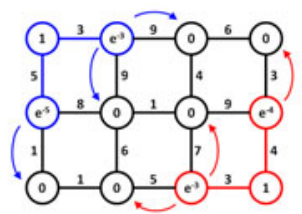

(b)

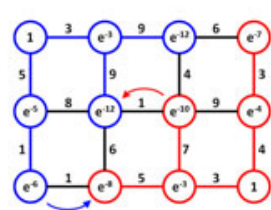

(c)

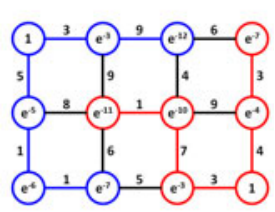

(d)

Fig. 1. (a) The graph is initialized with similarities as edge weights and vertex values 1 for seeds, 0 elsewhere; (b-c) intermediate propagation steps for CA; (d) shows the final vertex values obtained from $\mathrm{CA}$ which can also be obtained as the shortest path from each vertex to a seed

\subsection{Seed Selection Based on Tumor Response Measurement Criteria}

As each path, defining the labeling of a vertex ends at a seed, the efficiency of the algorithm can be increased by choosing the background seeds on a closed surface around the volume of interest (VOI) because the result of labeling inside the VOI is equivalent to using the whole data set.

Robustness to seed selection is an important property of a segmentation algorithm, as it is natural to expect similar results for the same tumor while allowing the user to guide the segmentation process interactively by imposing constraints in different way. In RECIST tumor response criteria [11, a general procedure to follow-up tumor progress is to measure the maximum observable tumor diameter. Our seed selection algorithm employs the same idea to follow the familiar clinical routine to which the clinicians are used to. Focusing on tumor segmentation problem, we utilize the following seed selection procedure (see Fig. 2ha, 2 $\mathrm{b}$ ):

- Ask user to draw a line along the maximum visible diameter of the tumor.

- Crop the line $15 \%$ from each end and thicken to 3 pixels wide to obtain fg seeds. 
- Choose bounding box of the sphere having $20 \%$ longer of the line as VOI.

- Use the 1 voxel wide border of this VOI as background seeds.

One obvious drawback is that the input seed information is obtained from only a single slice of the tumor volume, hence it is not guaranteed that the depth of the tumor will also coincide with the VOI. However, our experimental studies revealed that spherical assumption for the tumor is mostly valid.

\subsection{Adapting Transition Rule to Tumor Characteristics}

In the seeded tumor segmentation application for heterogeneous tumors, which mostly consist of a ring enhancing region around a dark necrotic core (and also irregular borders), most of the foreground seeds fall in the necrotic region. This causes the segmentation algorithm to get stuck at necrotic to active transition borders. To overcome such problems, a prior knowledge is added to the edge weight function as follows:

$$
w_{j k}=e^{-\beta_{\text {tumor }}^{l, \operatorname{sgn}\left(I_{j}-I_{k}\right)}\left\|I_{j}-I_{k}\right\|} \text { where sgn denotes sign function. }
$$

Enhancing tumor cells are brighter than the normal tissue, and more centrally located necrotic core is darker, hence by adjusting $\beta$ parameter, the weight reduction (strength loss) of a tumor state while passing through a ramp up gradient is adjusted to be lower than other cases:

$$
\beta_{\text {tumor }}^{l, \operatorname{sgn}\left(I_{j}-I_{k}\right)}=\left\{\begin{array}{l}
0.7 \text { if } l_{k} \text { is foreground and } \operatorname{sgn}\left(I_{j}-I_{k}\right)=+1 \\
1.0 \text { otherwise }
\end{array}\right.
$$

Although, some of the properties we derived for this algorithm is no more valid, and due to asymmetric edge values, we can no more interpret the algorithm in the undirected graph framework, our experimental results revealed that the new tumor CA (tCA) algorithm significantly improved the results obtained, especially on glioblastomas.

\subsection{Using Level Set on Strength Maps}

Smoothing is an important prior in segmentation of brain tumors from post contrast T1 images, because of three main reasons: First, an area surrounded by tumor tissue is considered as a tumor region even the intensity characteristics likely to be healthy. Secondly, it is possible to include misclassified necrotic regions to tumor region, which are usually surrounded by enhanced tissue. Finally, it is possible to exclude nearby structures such as arteries that are enhanced by adminstration of the contrast agent.

As described in Section 2.1, cellular automata algorithm assigns a label $l$, and a likelihood value $x_{i}$ in the interval $(0,1]$ to each voxel $v_{i}$. The latter indicates how much it is likely to assign one of the labels to the voxel. Remapping values of the final map $X=\left\{x_{i}\right\}_{i \in V}$ to the interval $(-1,1)$ for all voxels in $V$, we obtain a new map $M$ : 


$$
M_{i}= \begin{cases}\frac{x_{i}-\min (X)}{\max (X)-\min (X)} & \text { if } l_{i} \text { is foreground } \\ -\frac{x_{i}-\min (X)}{\max (X)-\min (X)} & \text { if } l_{i} \text { is background }\end{cases}
$$

with values $M_{i}$ at a voxel i. Finally, a level set snake is evolved on map $M$ with a piecewise constant region assumption of [2], however by using a local Gaussian kernel to define inner and outer regions around the propagating surface, to obtain the final tumor segmentation map.

Steps of the proposed cellular automata based tumor segmentation algorithm is shown in Fig. 2, First, the user draws a line over the largest visible diameter of the tumor (a); using this line, a VOI is selected with foreground-background seeds (b); tCA algorithm is run on the VOI to obtain a label map and strengths at each voxel (c); label maps and strengths are combined to obtain the signed strength values, i.e. map $M$, such that contours have value of zero (d). The map $\mathrm{M}$ is used to evolve a level-set snake. In (d), initial level set contour is depicted in white, and final evolved contour is shown in black. Comparison to expert segmentation (blue) is visualized in (e), overlayed with tCA result (red), and tCA-Level set result (yellow).

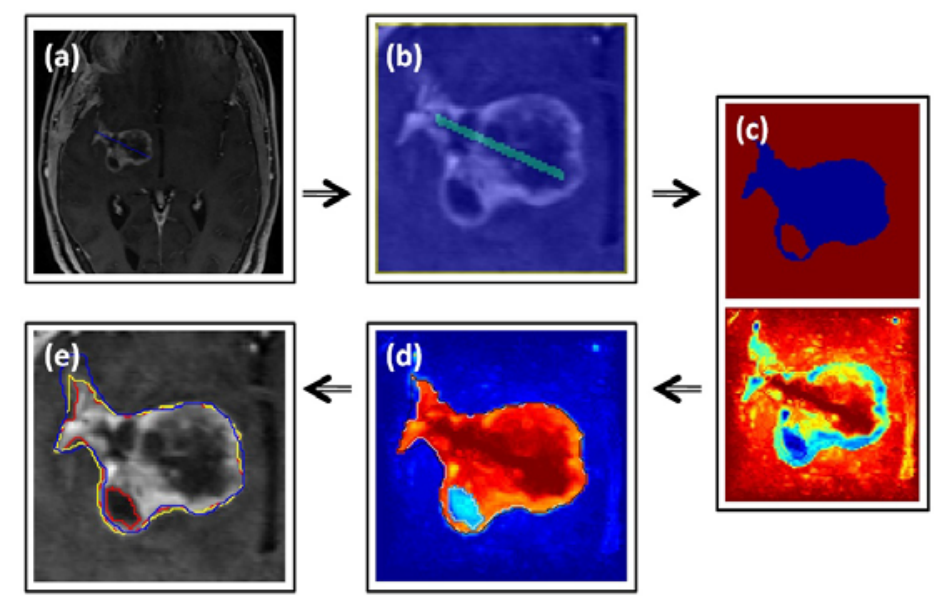

Fig. 2. Steps of the proposed tumor segmentation method: see text for explanations

\section{Results and Discussion}

An expert-segmentation during a radiation therapy planning session is compared against results of Graph Cut (GC), Random Walker (RW), Grow-cut, and the tCA over a slice (see Fig (3). It can be observed that highly varying necrotic and enhancing tumor characteristics present challenges to all computer algorithms, which fail to capture the expert segmented boundaries. Cellular-automata based algorithms grow-cut and tCA could propagate further towards the enhanced tumor margins. 


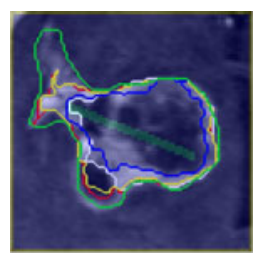

Fig. 3. Comparison of graph based algorithms: Expert in Green; RW in Blue (Dice: \%70), GC in White (Dice: \%80); Grow cut in Yellow (Dice: \%87), tCA in Red (Dice: \%89)
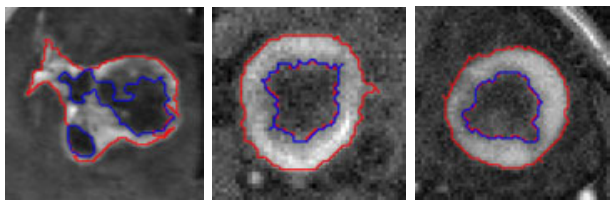

Fig. 4. Segmentation of enhancing and necrotic regions of the tumor using multilabel cellular automata

\subsection{Enhancing/Necrotic Core Segmentation Qualitative Results}

Cellular automata segmentation algorithm is applied on heterogeneous tumors with enhancing and necrotic regions, whose delineation is important especially in assessment of radiotherapy response. In the first step, tCA-LS method is applied to obtain a total tumor mask. Tumor and necrotic seeds are chosen by applying a threshold to intensity histogram of the segmented region. For background seeds, a one voxel boundary around the VOI is used. tCA algorithm is initialized with these 3 label seeds on a single slice of three tumors and the results are given in Fig 4 .

\subsection{Validations on Synthetic Data}

Dice similarity measure, $\operatorname{Dice}(A, B)=2 \times s(A \cap B) /(s(A)+s(B))$, is used to quantify the overlap between obtained segmentation maps and expert manual segmentations extracted from radiotherapy planning sessions for each tumor. To measure the robustness of the method, for each tumor case, overlap for 5 different initialization lines are calculated and mean and standard deviation of the overlap are given, and the performance is compared between GC, Grow-cut, tCA, tCA-LevelSet(LS) 1 .

Five synthetic brain tumor datasets, available online from University of Utah 2 are used for validation and the dice measures are reported in Table 1. Synthetic Tumor 5, which is not enhanced with contrast agent and out of scope of the proposed algorithm, is included for the completeness of the Utah dataset (see Fig $5 \mathrm{~s}$ ).

\subsection{Validations on Tumors That Undergo Radiation Therapy Planning}

Validations on clinical data set were carried out over high resolution $(\approx$ $0.5 \times 0.5 \times 1.0 \mathrm{~mm})$ post Gd T1 weighted 3D FLASH MRI scans of 13 tumors of

\footnotetext{
${ }^{1}$ Due to unavailability of RW method in $3 \mathrm{D}$, it was not included in the validation tests.

${ }^{2}$ http://www.ucnia.org/softwaredata/5-tumordata/10-simtumordb.html
} 
Table 1. Dice overlap \pm std deviations over 5 different initial seed lines for each tumor for synthetic tumor data set from 12 ]

\begin{tabular}{|l|c|c|c|c|}
\hline & Graph cut & Grow cut & tCA & tCA-Level Set \\
\hline Synthetic Tumor 1 & $6.6 \pm 2.5$ & $83.8 \pm 1.1$ & $87.4 \pm 0.9$ & $90.4 \pm 0.7$ \\
Synthetic Tumor 2 & $58.0 \pm 32.3$ & $77.8 \pm 3.2$ & $81.4 \pm 3.6$ & $84.6 \pm 4.4$ \\
Synthetic Tumor 3 & $96.5 \pm 0.0$ & $96.2 \pm 0.3$ & $96.3 \pm 0.3$ & $97.6 \pm 0.2$ \\
Synthetic Tumor 4 & $91.1 \pm 0.7$ & $89.3 \pm 1.0$ & $91.9 \pm 0.8$ & $93.0 \pm 0.9$ \\
Synthetic Tumor 5 & $11.6 \pm 7.4$ & $73.6 \pm 2.7$ & $73.1 \pm 3.3$ & $69.1 \pm 5.7$ \\
\hline Average Overlap & $52.8 \pm 42.5$ & $84.1 \pm 9.0$ & $86.0 \pm 9.1$ & $86.9 \pm 11.0$ \\
\hline
\end{tabular}

Table 2. Dice overlap \pm std deviations over 5 different initial seed lines for each tumor demonstrate improved overlap with the proposed method

\begin{tabular}{|l|l|c|c|c|c|}
\hline & & Graph cut & Grow cut & tCA & tCA-Level Set \\
\hline Tumor 1 & Metastasis & $76.8 \pm 0.0$ & $79.5 \pm 2.0$ & $80.2 \pm 1.6$ & $83.5 \pm 0.3$ \\
Tumor 2 & Gliosarcoma; Grade IV & $15.0 \pm 5.5$ & $53.5 \pm 7.4$ & $57.6 \pm 6.0$ & $69.8 \pm 5.5$ \\
Tumor 3 & Grade II Astrocytoma & $34.5 \pm 16.0$ & $76.9 \pm 3.1$ & $83.2 \pm 1.0$ & $89.1 \pm 1.2$ \\
Tumor 4 & Metastasis & $17.0 \pm 37.1$ & $72.6 \pm 5.8$ & $74.6 \pm 4.0$ & $79.5 \pm 3.2$ \\
Tumor 5 & Metastasis & $39.0 \pm 6.5$ & $44.4 \pm 5.1$ & $46.5 \pm 3.0$ & $51.5 \pm 2.6$ \\
Tumor 6 & Metastasis & $5.1 \pm 8.6$ & $51.7 \pm 5.3$ & $54.6 \pm 4.9$ & $60.5 \pm 3.7$ \\
Tumor 7 & Metastasis & $76.6 \pm 2.5$ & $73.8 \pm 1.9$ & $74.8 \pm 1.5$ & $81.3 \pm 1.8$ \\
Tumor 8 & Metastasis & $69.3 \pm 0.3$ & $76.6 \pm 0.9$ & $76.9 \pm 1.0$ & $81.6 \pm 0.9$ \\
Tumor 9 & Metastasis & $55.3 \pm 1.9$ & $63.3 \pm 5.1$ & $65.2 \pm 4.2$ & $68.4 \pm 4.0$ \\
Tumor 10 & Meningioma & $71.6 \pm 10.0$ & $61.1 \pm 6.8$ & $65.5 \pm 6.3$ & $76.9 \pm 3.9$ \\
Tumor 11 & Meningioma & $83.0 \pm 0.1$ & $69.8 \pm 3.4$ & $73.1 \pm 2.7$ & $83.5 \pm 1.3$ \\
Tumor 12 & Meningioma & $44.9 \pm 24.5$ & $49.0 \pm 8.8$ & $52.7 \pm 7.5$ & $64.1 \pm 5.9$ \\
Tumor 13 & Meningioma & $68.6 \pm 1.7$ & $67.7 \pm 1.6$ & $68.0 \pm 1.8$ & $71.7 \pm 1.8$ \\
\hline Average Overlap & & $50.5 \pm 26.5$ & $64.6 \pm 11.7$ & $67.1 \pm 11.4$ & $74.0 \pm 10.8$ \\
\hline
\end{tabular}

7 patients obtained from Anadolu Medical Center. As the ground truth for segmentation, we used the tumor contours outlined manually by a radio-oncologist for radiotherapy planning. The clinical classification of tumors along with the segmentation performances are tabulated in Table 2 ,

The results we observed with the GC approach exhibit similar problems reported before in [7] such as shrinking bias due to minimum cut optimization. The shortest path algorithms, equivalently CA, showed lack of the shrinking bias problem. The proposed tCA-LS algorithm exhibit a lower coefficient of variation (std/mean) on the average compared to the other methods used in validation.

\subsection{Qualitative Results}

We present qualitative results of both synthetic and real tumors using the proposed tCA-LS algorithm in Figure 5. The result on a synthetic tumor with a non-enhanced region having no boundary to healthy tissue is given in Fig 5(e). The metastasis (Tumor 6) in Fig [5) is a small tumor (1.4cc) with weak boundaries, which produces a low overlap score even for small errors on the boundaries. 
The synthetic tumor in Fig 5)(g) is not enhanced by the contrast agent, and the result obtained leaks outside due to the lower intensities than surrounding tissue and weak boundaries. For the metastasis in Fig 5(h), surrounding bright tissue is misclassified as tumor, even after smoothing with a level set.

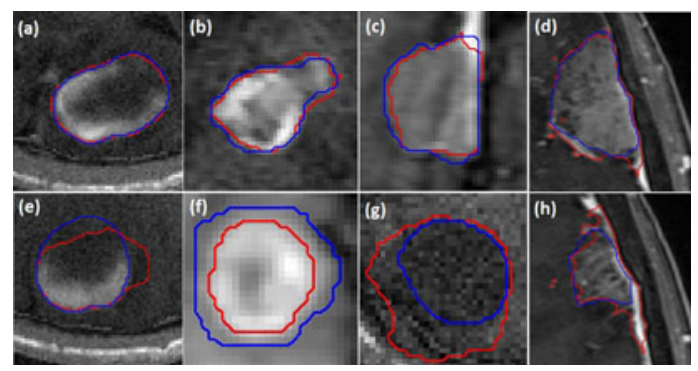

Fig. 5. Examples of typical (top row) and challenging cases (bottom row) obtained by tCA-LS: Expert segmentation in Blue, tCA-LS in Red

\section{Conclusion}

The proposed segmentation algorithm for the problem of tumor delineation, has only two main parameters: $\beta_{\text {tumor }}^{l,+}, l \in\{f g, b g\}$ and mean curvature term weight in the level set evolution. One future work includes optimizing both curvature term and the tumor sensitivity parameter $\beta_{\text {tumor }}^{l}$ over a larger tumor database, although the results over 18 tumors of varying degrees showed that the algorithm performs with high overlap ratios even with the fixed heuristic values. Another item is to investigate the issues related to the VOI and seed selection procedure. Our current work includes assessment of the tumor response to therapy, which is built on the given segmentation framework.

Acknowledgement. This work was partially supported by TUBITAK Grant No:108E126, and EU FP7 Grant No: PIRG03-GA-2008-231052.

\section{References}

1. Zou, K.H., Warfield, S.K., Bharatha, A., Tempany, C.M.C., Kaus, M.R., Haker, S.J., Wells, W.M., Jolesz, F.A., Kikinis, R.: Statistical validation of image segmentation quality based on a spatial overlap index. Academic Radiology 11(2), 178-189 (2004)

2. Chan, T.F., Vese, L.: Active contours without edges. IEEE Transactions on Image Processing 10(2), 266-277 (2001)

3. Ho, S., Bullitt, E., Gerig, G.: Level-set evolution with region competition: Automatic 3-d segmentation of brain tumors. In: ICPR, vol. 1, p. 10532 (2002)

4. Liu, J., Udupa, J.K., Odhner, D., Hackney, D., Moonis, G.: A system for brain tumor volume estimation via $\mathrm{mr}$ imaging and fuzzy connectedness. Computerized Medical Imaging and Graphics 29, 21-34 (2005) 
5. Boykov, Y., Jolly, M.P.: Interactive graph cuts for optimal boundary and region segmentation of objects in n-d images. In: ICCV, pp. 105-112 (2001)

6. Grady, L.: Random walks for image segmentation. PAMI 28(11), 1768-1783 (2006)

7. Couprie, C., Grady, L., Najman, L., Talbot, H.: Power watersheds: A new image segmentation framework extending graph cuts, random walker and optimal spanning forest. In: ICCV (2009)

8. Sinop, A., Grady, L.: A seeded image segmentation framework unifying graph cuts and random walker which yields a new algorithm. In: ICCV (2007)

9. Szeliski, R., et al.: A comparative study of energy minimization methods for markov random fields with smoothness-based priors. PAMI 30(6) (2008)

10. Vezhnevets, V., Konouchine, V.: Growcut - interactive multi-label n-d image segmentation by cellular automata. In: Graphicon, Novosibirsk Akademgorodok, Russia (2005)

11. Therasse, P.: Evaluation of response: new and standard criteria. Annals of Oncology 13, 127-129 (2002)

12. Prastawa, M., Bullitt, E., Gerig, G.: Synthetic ground truth for validation of brain tumor mri segmentation. In: Duncan, J.S., Gerig, G. (eds.) MICCAI 2005. LNCS, vol. 3749, pp. 26-33. Springer, Heidelberg (2005) 